\title{
AxCELL: Automatic Extraction of Results from Machine Learning Papers
}

\author{
Marcin Kardas ${ }^{1}$ \\ Piotr Czapla ${ }^{2}$ \\ Pontus Stenetorp 3
}

\author{
Sebastian Ruder ${ }^{4} \quad$ Sebastian Riedel $^{1,3}$ \\ Ross Taylor ${ }^{1}$ \\ ${ }^{1}$ Facebook AI Research, \{mkardas, sriedel, rjt, rstojnic $\} @$ fb.com
${ }^{2}$ n-waves, piotr.czapla@n-waves.com
${ }^{3}$ University College London, p.stenetorp@cs.ucl.ac.uk
${ }^{4}$ DeepMind, ruder@google.com
}

Robert Stojnic ${ }^{1}$

\begin{abstract}
Tracking progress in machine learning has become increasingly difficult with the recent explosion in the number of papers. In this paper, we present AxCELL, an automatic machine learning pipeline for extracting results from papers. AXCELL uses several novel components, including a table segmentation subtask, to learn relevant structural knowledge that aids extraction. When compared with existing methods, our approach significantly improves the state of the art for results extraction. We also release a structured, annotated dataset for training models for results extraction, and a dataset for evaluating the performance of models on this task. Lastly, we show the viability of our approach enables it to be used for semi-automated results extraction in production, suggesting our improvements make this task practically viable for the first time. Code is available on GitHub 1
\end{abstract}

\section{Introduction}

Machine learning studies how machines learn with respect to a task, a performance metric, and a dataset (Mitchell, 2006). The (task, dataset, metric name, metric value) tuple can therefore be seen as representing a single result of a machine learning paper. To make progress as a field we need to make comparisons between results achieved with different methodologies. In light of the explosion in the number of machine learning publications in recent years, such comparisons have become more difficult $\left.\right|^{2}$ This poses serious challenges to peer review, among others. For instance, across ten language modelling papers submitted to ICLR 2018, the perplexity score of the best baseline differed by more

${ }^{1}$ https://github.com/paperswithcode/ axcell

${ }^{2}$ In 2019, over 33,000 machine learning papers were published on the arXiv.org open-access e-print archive, with a year-on-year growth of around $50 \%$ since 2015. than 50 points (Ruder, 2018).

One way to deal with the deluge of papers is to develop automatic approaches for extracting results from papers and aggregating them into leaderboards. Authors typically publish their results in a tabular format in the paper, including a selection of comparisons between their approach and past papers. Automatic extraction of result tuples from tables-and optionally metadata such as model names - enables a full comparison between published methods.

Online leaderboards for comparison have become increasingly common in the research community. But these are only available for a few tasks and do not aid the comparison of models across tasks. To fill the gap, result aggregation tools such as Papers With Code $\unlhd^{3}$ and NLP-Progress $4^{4}$ utilise crowdsourced community contributions to populate paper leaderboards. However, human annotation of results can be laborious and error-prone, leading to omission or misreporting of paper results. Automating at least some parts of the process can speed-up the annotation, reduce number of errors and lower the expert knowledge required to correctly annotate a paper. This motivates the need for a machine learning approach to create a comprehensive results resource for the field.

Existing state-of-the-art approaches for results extraction are brittle and noisy, relying on text formatting hints and tables extraction from PDF files (Hou et al., 2019). In contrast, we propose AxCELL, a pipeline for automatic extraction of results from machine learning papers. AXCELL breaks down the results extraction task into several subtasks including table type classification, table semantic segmentation and linking results to leaderboards. We employ an ULMFiT-based classifier

3 https: / / www . paperswithcode.com/sota http://nlpprogress.com/ 
architecture (Howard and Ruder, 2018) to make full use of paper and table context to interpret tabular content, and extract results accordingly.

As a whole, this paper makes three main contributions to the literature. First, we significantly improve over the state-of-the-art for results extraction with our AXCELL system. On the subset of the NLP-TDMS dataset of Hou et al. (2019) where ${ }_{\text {LT }} \mathrm{X}$ code is available, our approach achieves a micro $F_{1}$ score of 25.8 compared to the state of the art of 7.5. Secondly, we release a structured, annotated dataset for training models for results extraction, and an evaluation dataset for evaluating the performance of models on this task. Lastly, our approach is used in an in-production setting at paperswithcode.com to semi-automatically (by aiding the human review) extract results from papers and track progress in machine learning.

\section{Related Work}

Results Extraction. Previous works have studied the problem of extracting results tuples (task, dataset, metric name, metric value) from papers. Singh et al. (2019) perform search over publications and compose a leaderboard for a queried triplet. Similar to our approach, they use tables extracted from $\mathrm{LT}_{\mathrm{E}} \mathrm{X}$ sources. In contrast, they do not extract absolute metric values but rank papers and do not appear to utilise the text content of publications. Our goal in this paper is to extract complete results to create leaderboards, so unlike Singh et al. (2019), we focus on extracting raw metric values. Additionally we make use of the content of the publication as context for entity recognition and linking.

Closer to our formulation, Hou et al. (2019) extract absolute metric values alongside the metric name, task and dataset. They also use text excerpts as well as direct tabular information to make inferences for table contents. They frame extraction as a natural language inference problem and apply an NLI model based on a BERT architecture (Devlin et al. 2019) to extract results from PDF files. The disadvantage of this approach is that using PDFs leads to a lot of noise in structural information such as the partition of a table into cells. In our work, we explicitly utilise the structural information from the IATEX source to extract entire tables in order to perform semantic segmentation. We demonstrate that this structural information and segmentation are crucial for boosting extraction performance.
Table Extraction. The more general problem of retrieving information from tables has been studied in past works (Milosevic et al., 2019; GhasemiGol and Szekely, 2018; Wei et al., 2006; Herzig et al., 2020). Our focus in this paper is on the problem of extracting and interpreting content of tables characteristic to machine learning papers. The goal of our table semantic segmentation model is to classify cells into categories. That is, instead of performing structural segmentation where one tries to distinguish between captions, headers and rows in a stream of text (Pinto et al., 2003) we focus on semantic segmentation (i.e., assigning roles to each cell) of tables.

\section{Our Approach}

The task of paper results extraction is to take a machine learning paper as an input and extract results contained within the paper, specifically tuples of the form (task, dataset, metric name, metric value). As an example, if we were to take the EfficientNet paper of Tan and Le (2019) as an input, some example results tuples we would want to extract would be (Image Classification, ImageNet, Top 1 Accuracy, 84.4\%), (Image Classification, ImageNet, Top 5 Accuracy, 97.1\%) and (Image Classification, Stanford Cars, Accuracy, 94.7\%).

To tackle this problem effectively we define subtasks that take us from paper to results. In particular, we introduce the AxCELL pipeline that consists of the following subtasks: (i) table type classification, identifying whether a table in a paper has relevant results; (ii) table segmentation, segmenting and classifying table cells according to whether they hold metrics, datasets, models, etc.; and (iii) linking results to leaderboards, taking the result tuples and matching them to an existing leaderboard of results. The end-to-end system is shown in Figure 1 with reference to an example. We now introduce the different components of AXCELL.

\subsection{Table Type Classification}

The first stage of AXCELL is to categorize tables from papers into one of three categories: leaderboard tables, ablation tables and irrelevant tables. A leaderboard table contains the principal results of the paper on a selected benchmark, including comparisons with other papers. An ablation table compares different permutations of the paper's methodology. 


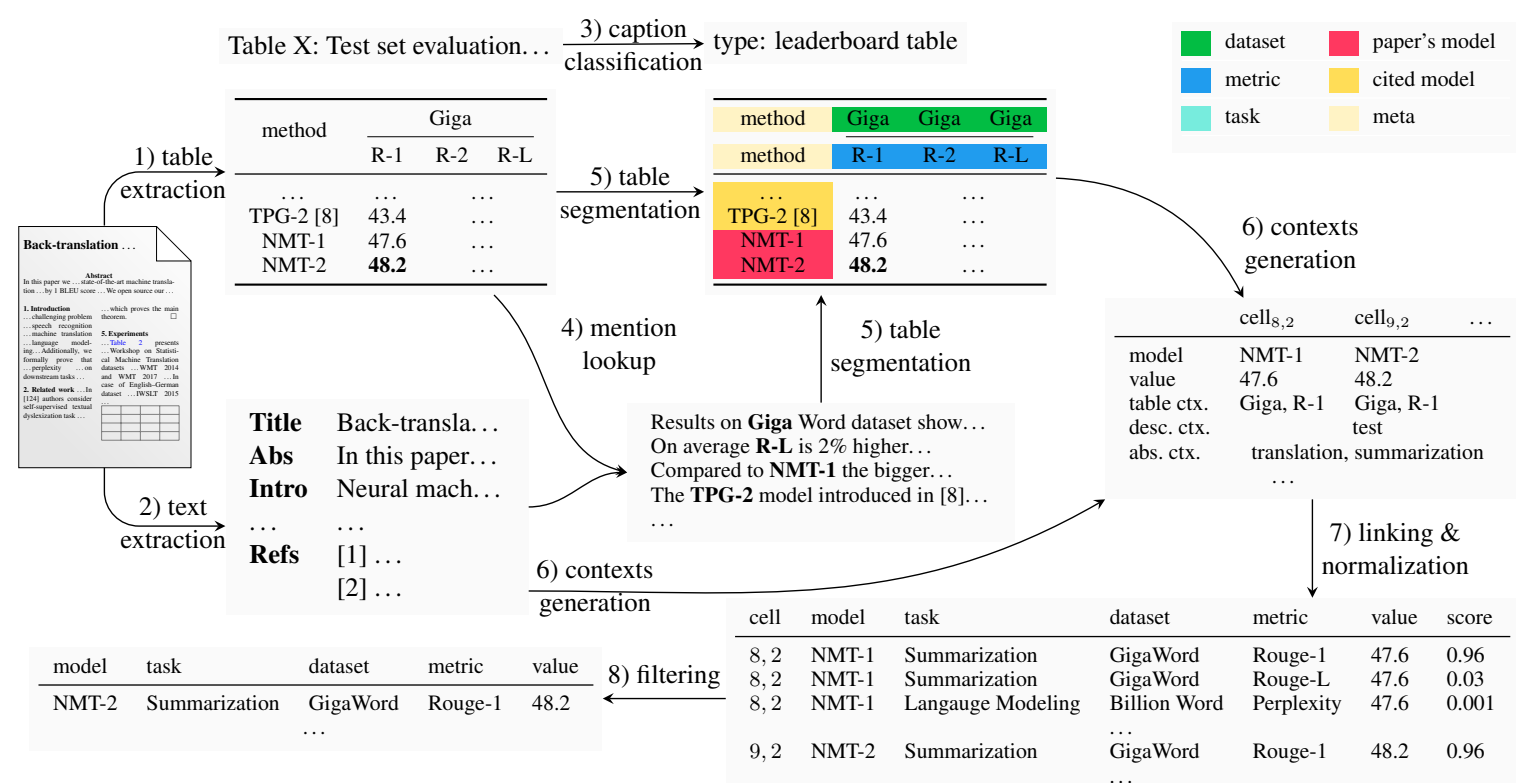

Figure 1: Graphical depiction of AXCELL. The extraction starts with $\mathrm{HT}_{\mathrm{E}} \mathrm{X}$ source code of a paper, from which we extract 1) tables and 2) text. 3) We classify the caption to filter out irrelevant tables. 4) The content of each cell is looked up in the paper's text. Retrieved mentions are used to 5) segment cells based on their meaning (see the legend in the top-right corner). The segmented table and the paper's text are used to 6) obtain contexts for each numeric cell. 7) Results tuples are scored based on contexts and numeric values are normalized to match required format. 8) Inferior results or results below a confidence threshold are filtered out.

Lastly, irrelevant tables include hyperparameters, dataset statistics and other information that is not directly relevant for result extraction.

For this stage we employ a classifier with a ULMFiT architecture (Howard and Ruder, 2018) with LSTM layers and a SentencePiece unigram model (Kudo, 2018) for tokenization 5 We train the SentencePiece model and pretrain a left-to-right ULMFiT language model on text of papers from an unlabelled dataset of arXiv articles (see Section 4). Table 5 in the Appendix contains details on the hyperparameters and training regime 6

The classifier head is a standard ULMFiT classifier with a pooling layer followed by two linear layers. We treat the problem as a two-label classification with labels: leaderboard and ablation. A table is considered irrelevant if it is neither a leaderboard nor ablation (we use a confidence threshold of 0.5). In practice it is common for a single table to include both principal results introduced in a given paper as well as results of ablation

${ }^{5}$ Our classifier uses the fast.ai implementation (Howard and Gugger. 2020).

${ }^{\circ} \mathrm{We}$ experimented with finetuning alternative language models such as BERT and SciBERT but our initial experiments did not yield superior results. A full investigation of alternative models, including pretraining from scratch, is left for future research. studies. For this reason we extract results from both leaderboard and ablation tables and pick only the best results during filtering (see Section 3.6). We train the model on the SEGMENTEDTABLES dataset (see Section 4.2).

\subsection{Table Segmentation}

The second stage of AXCELL is to pass relevant tables to a table segmentation subtask. The goal is to annotate each non-numeric cell of a table with a label denoting what type of data a given cell contains. To this end, we classify each table cell into one of: dataset name, metric name, paper model, cited model, and other (containing meta and task cells). An example of a segmented table is shown in Figure 1

To help classify each table cell, we provide a context in which the cell content is mentioned. We search for cell content in the full paper content using a BM25 scoring algorithm. Retrieved text fragments are then passed to a ULMFiT-based classifier with some handcrafted features for the cell. These features include information such as the position of the cell in the table, whether the cell is a header, and cell styles. A full list is available in the Appendix. For processing the retrieved text fragments, the retrieved term from the cell is replaced 
On TREC-6, <MASK> significantly

improves upon training from

scratch; as examples are shorter

and fewer, supervised and

semi-supervised <MASK> achieve

similar results.

Figure 2: An example of a text excerpt from the paper by Howard and Ruder (2018) used as evidence for a cell content query with ULMFiT (covered with $<$ MASK $>$ token) as paper model.

with a special mask $<$ MASK $>$ token to inhibit memorization of common names (see Figure 2 for an example). Table segmentation can then be treated as a classification problem with 5 exclusive labels. We use the same pre-trained language model to train the table type classifier. Results for this stage of the model are outlined in Table 3 .

\subsection{Cell Context Generation}

The next stage after table segmentation is to generate contexts for numeric cells. As an example, if we know a numeric cell has a dataset cell somewhere in its row, and a model cell somewhere in its column, then this table context is informative for deciding the dataset and model for this result. But there is much broader context in the paper that is useful for linking.

For example, a paper studying semantic segmentation with models evaluated on KITTI and CamVid datasets could mention semantic segmentation in the introduction, test set in a subsection referring to a results table, KITTI in the description of that table and class IoU in the column header. Figure 3 shows a visual representation of this hierarchy of context.

To reflect this hierarchy we generate several types of contexts for each cell. The table context, as discussed, looks at a numeric cell and other cells in its row or column labeled as model, dataset or metric. We also define text contexts: a caption context, the table caption; a mentions context, text fragments referencing the table; an abstract context, the paper abstract; and a global paper context, containing the entire paper text. The gathered contexts are then used to link potential results to predefined leaderboards of results.

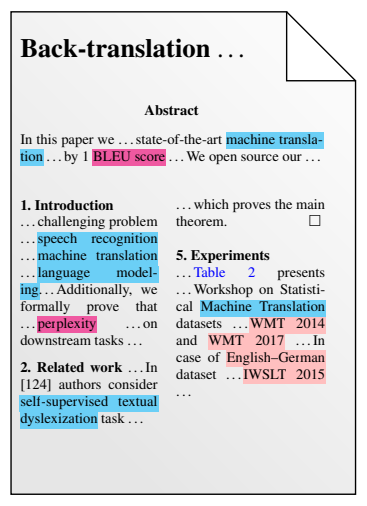

Table I: ... test set. . BLEU metric.

\begin{tabular}{cccc}
\hline & \multicolumn{2}{c}{ WMT 2014} & $\ldots$ \\
$\ldots$ & en-fr & fr-en & $\ldots$ \\
\hline$\ldots$ & $\ldots$ & $\ldots$ & $\ldots$ \\
NMT (ours) & $\mathbf{5 6 . 3}$ & 41.8 & $\ldots$ \\
\hline
\end{tabular}

Linking result:

Task: Machine Translation

Dataset: WMT2014 English-French Test

Metric: BLEU score

Value: 56.3

Model: NMT

Confidence: 0.98

Figure 3: Using the context hierarchy and evidences for linking. This figure highlights the context hierarchy, from the global paper to the specific table, the evidence for tasks (blue), datasets (pink) and metrics (violet) for the 56.3 value extracted from cell contexts, and lastly the result from linking.

\subsection{Linking Cells to Leaderboards}

Once we have the cell contexts, the next stage of AXCELL is to link them to leaderboards to form performance records. The goal is to take a metric value associated with a paper model cell and infer the leaderboard it is connected to. A leaderboard is defined by a (task, dataset, metric name) triplet. For example: (Image Classification, ImageNet, Top 1 Accuracy) can capture papers that report performance on Image Classification for ImageNet and report Top 1 Accuracy. To simplify the problem, we assume a closed-domain with all leaderboards known in advance. To match results to leaderboards we look for evidence in cell contexts, which we now explain.

Pieces of evidence are words or phrases that correspond to a task, dataset or metric. For example, SST-2, binary and polarity could all serve as evidence for the two-class Stanford Sentiment Treebank dataset (Socher et al., 2013). Pieces of evidence allow us to infer whether an entity has been mentioned in a given context. Using the same example, if "SST-2" appears in the table caption then this is evidence that a numeric value in the table could be linked to the Stanford Sentiment Treebank dataset.

\subsection{Model}

Our goal is to determine the probability $p(k \mid E)$ of a leaderboard $k \in\{1 \ldots K\}$ being associated with a given cell, conditioned on the evidence $E$ we 
have collected for this cell. Instead of modelling this directly using a discriminative model, we opt for a simple generative model $p(k, E)$ that can be adapted to new leaderboards as well as types of evidence without additional training data. While this should be possible for discriminative models as well, we leave this open for future work.

Let $E=\left\{e_{1}, \ldots, e_{s}\right\}$ consist of pieces of evidence $e_{j}$ of the form $e_{j}=(m, t, c)$ where $m$ is a mention such as "acc", $t$ is a type of entity such as "Metric" and $c$ is the type of context the evidence was found in, such as "Table." Our model generates leaderboard and evidence using $p(k, E)=p(k) p(E \mid k)$. To model the likelihood of evidence, we make a Naive Bayes assumption and set $p(E \mid k)=\prod_{e \in E} p(e \mid k)$.

We assume that the type of context $c$ controls the generation of the remainder of the evidence $m$ and $t$ :

$$
p(e \mid k)=p(m, t, c \mid k)=p(c) p(m, t \mid c, k) .
$$

Once we know the context type $c$, using a latent noise variable $n$ we generate evidence either independent or dependent of the actual leaderboard:

$$
\begin{aligned}
p(m, t \mid c, k) & =p(t \mid c, k)[p(n \mid t, c) p(m \mid n, t) \\
& +(1-p(n \mid t, c)) p(m \mid \neg n, t, k)] .
\end{aligned}
$$

Finally, we assume that a leaderboard generates its mention as follows:

$$
p(m \mid \neg n, t, k)=p(m \mid \neg n, t, \operatorname{property}(t, k))
$$

where property $(t, k)$ is the $t$ property of the leaderboard $k$. For example if the leaderboard $k$ consists of (Image Classification, ImageNet, Accuracy) then $\operatorname{property}($ Metric, $k)=$ Accuracy .

Inference To score a leaderboard $k$ given evidence $E$, we calculate $p(k, E) / \sum_{k^{\prime}} p\left(k^{\prime}, E\right)$ summing over all leaderboards in the taxonomy. This is feasible as we assume a closed-domain scenario.

Estimation Most of our parameters are handset to uniform distributions. In particular, we set $p(k)=\frac{1}{K}, p(t \mid c, k)=\frac{1}{3}, p(c)=\frac{1}{5}$. We set $p(m \mid \neg n, t, \operatorname{property}(t, k))$ to be inversely proportional to the number of other entities of type $t$ with the same mention evidence $m$ (see Appendix $\mathrm{C}$ for details).

The probabilities $p(n \mid t, c)$ of a mention of type $t$ in context $c$ being noisy are tuned manually for each of $15(t, c)$ pairs. The probabilities $p(m \mid n, t)$ of a noisy mention are assumed to be the same for all mentions of a given type $t$ and are tuned as well. We tune 18 parameters in total.

\subsection{Filtering}

The final step of AXCELL is to filter out (i) results for cited models, (ii) results with a linking score that is too low and (iii) inferior results (to avoid extraction of ablation results).

First, we filter out records not associated with models introduced in a paper being processed. We then remove records for which a linking score is below some given threshold. The remaining records are grouped by leaderboard and for each leaderboard only the best result is kept, based on higher is better annotation available in taxonomy; e.g., Accuracy would keep higher values, Error Rate would keep lower values. Finally, we remove all results with a linking score below the second threshold. This gives us the final list of results tuples extracted from the paper.

\section{Dataset}

In this section we explain the datasets we used for training and evaluating AXCELL for results extraction. The primary input we use for a training dataset is $\mathrm{IAT}_{\mathrm{E}} \mathrm{X}$ source code of machine learning papers from arXiv.org. Over $90 \%$ of considered papers have source code available. This allows us to obtain a high quality dataset without common artifacts that arise from extracting data directly from PDF files.

For training our models we use two main datasets:

- ArXivPapers: An unlabelled dataset of over 100,000 machine learning papers. Used for language model pre-training.

- Segmentedtables: A table segmentation dataset where each cell is annotated according to whether it is a paper, metric, dataset, and so on. Used for table segmentation and table type classification.

We manually tune the linking and filtering performance of our method using a validation dataset:

- LiNKEDRESULTS: An annotated dataset of over 200 papers with results tuples, capturing the performance of models in the papers, and links to tables. 
Lastly we evaluate the end-to-end performance of AXCELL on our test set:

- PWC LEAdERboARds: An annotated dataset of over 2,000 leaderboards with results tuples. Used for end-to-end performance evaluation.

We now describe in detail these datasets.

\section{1 arXiv Papers}

The dataset contains 104, 710 papers published on arXiv.org between 2007-2020. 93, 811 papers are available with $\mathrm{IET}_{\mathrm{E}} \mathrm{X}$ sources, from which we extracted 277,946 tables in total. Due to licensing limitations the dataset we release with this paper contains only metadata (available in the public domain) and links to articles. The dataset is unlabeled, designated for use in self-supervised pretraining.

\subsection{Segmented Tables}

This is a dataset for table classification and segmentation, containing 1994 annotated tables from 352 articles. The dataset provides data on dataset mentions in captions, the type of table (leaderboard, ablation, irrelevant) and ground truth cell annotations into classes: dataset, metric, paper model, cited model, meta and task.

\subsection{Linked Results}

This is a set of 239 papers we annotated with 1591 results tuples, capturing the performance of models in the papers. Additionally we include metrics scores in a normalized form. We also record metadata such as the names of the models used in papers. Each results tuple (task, dataset, metric name, metric value) is linked to a particular table, row and cell it originates from. Note that results that appear outside of a table, for instance in the paper's text or graphs, are not present in this dataset.

\subsection{PWC Leaderboards}

This is a dataset of 2,291 leaderboards, where the data is collected from the Papers with Code labelling interface (see Figure 5 in Appendix). This interface allows annotators on Papers with Code to take a paper and label it with results tuples. Annotations are then reviewed by the community and revised if necessary. Since this is the biggest and most diverse curated ground-truth dataset, it is a good test for evaluating the end-to-end performance of our solution.
Table 1: End-to-end extraction results on subset of NLP-TDMS (Exp) dataset.

\begin{tabular}{lccccccc}
\hline \multirow{2}{*}{ Method } & \multicolumn{3}{c}{ Micro } & & \multicolumn{3}{c}{ Macro } \\
\cline { 2 - 3 } \cline { 5 - 7 } & $\mathrm{P}$ & $\mathrm{R}$ & $\mathrm{F}_{1}$ & $\mathrm{P}$ & $\mathrm{R}$ & $\mathrm{F}_{1}$ \\
\hline \multicolumn{4}{c}{ (task, dataset, metric) } \\
\hline TDMS-IE & 53.4 & 66.3 & 59.2 & 57.1 & 66.1 & 58.5 \\
AxCELL & 65.8 & 58.5 & 61.9 & 56.0 & 55.8 & 54.1 \\
\hline \multicolumn{6}{c}{ (task, dataset, metric, score) } \\
\hline TDMS-IE & 6.8 & 8.4 & 7.5 & 8.6 & 9.5 & 8.8 \\
AXCELL & 27.4 & 24.4 & 25.8 & 20.2 & 20.6 & 19.7 \\
\hline
\end{tabular}

\section{Experiments}

We now evaluate the end-to-end performance of AXCELL on the results extraction task. We evaluate on two datasets: the NLP-TDMS dataset introduced in Hou et al. (2019), in order to compare our method to the state of the art, and on our PWC LEADERBOARDS dataset, which contains many more leaderboards and acts as a more challenging benchmark.

\subsection{NLP-TDMS Results}

We compare AXCELL to the TDMS-IE model from Hou et al. (2019) on the NLP-TDMS dataset in Table 1 . The NLP-TDMS (Full) dataset contains 332 papers related to Natural Language Processing with 848 performance annotations of task, dataset, metric and score and 168 unique leaderboards. The subset NLP-TDMS (Exp) is limited to 77 leaderboards appearing in at least 5 papers. See Table 10 in the Appendix for dataset statistics. To compare with Hou et al. (2019), we use the Exp dataset.

Hou et al. (2019) extract records directly from PDF, so the methods are not fully comparable. In order to run AXCELL on that dataset we limit the dataset to papers for which $\mathrm{LTT}_{\mathrm{E}} \mathrm{X}$ source code is available. Table 1 shows results on that subset with TDMS-IE performance computed based on published predictions. Our solution yields significantly better results for whole records retrieval despite not being trained on their taxonomy (i.e., the zero-shot scenario in Hou et al. (2019)).

\subsection{PWC LEADERBOARDS Results}

Having validated the performance of our approach compared to the state of the art, we now apply it to our much larger dataset of leaderboards. Compared 
Table 2: Extraction results of AxCELL on PWC LEADERBOARDS dataset (restricted to our taxonomy) for entire records (TDMS), records without score (TDM) and individual entities.

\begin{tabular}{lccccccc}
\hline \multirow{2}{*}{ Entity } & \multicolumn{3}{c}{ Micro } & & \multicolumn{3}{c}{ Macro } \\
\cline { 2 - 5 } \cline { 7 - 8 } & $\mathrm{P}$ & $\mathrm{R}$ & $\mathrm{F}_{1}$ & & $\mathrm{P}$ & $\mathrm{R}$ & $\mathrm{F}_{1}$ \\
\hline TDMS & 37.4 & 23.2 & 28.7 & & 24.0 & 21.8 & 21.1 \\
\hline TDM & 67.8 & 47.8 & 56.1 & & 47.9 & 46.4 & 43.5 \\
Task & 70.6 & 57.3 & 63.3 & & 60.7 & 62.6 & 59.7 \\
Dataset & 70.2 & 48.4 & 57.3 & & 53.5 & 52.7 & 49.9 \\
Metric & 68.8 & 58.5 & 63.3 & 58.4 & 60.4 & 56.5 \\
\hline
\end{tabular}

to the NLP-TDMS dataset, whose taxonomy consists of 77 leaderboards, our taxonomy consists of 3,445 leaderboards making prediction much more challenging.

The results of our approach for extracting each entity are detailed in Table 2. We achieve reasonable performance on extracting the full TDMS (task, dataset, metric, score) tuple, which is the most challenging setting and the highest scores for extracting task and metric information. The lower scoring entities are generally the ones that depend on the quality of extraction of other entities. For example, extracting leaderboards depends on how well we extract task, dataset and metric entities.

The large difference in performance between extraction of TDM and full TDMS tuples is due to the fact that in order to get the score right, the model needs to correctly predict the table, column and row the score value is present in. Additionally, the extracted value needs to be normalized. On the other hand, the right TDM can often be inferred from other results reported in a paper.

\section{Performance Studies}

Due to working with machine learning papers from multiple domains (from CV to NLP to biology) and a multistep approach (where errors compound) the errors are characterized by a long-tail distribution and it is difficult to pin-point the biggest source of errors. In this section, we analyze the various steps of AXCELL in order to better understand their relative importance.

\subsection{Table Type Classification}

The biggest issue of table type classification is in distinguishing between leaderboard and ablation tables (see Figure 7 in Appendix). These tables can be very similar structurally: ablations may even compare on the same split of data as the primary result. As the distinction is not always clear, during results retrieval we extract results from both types of tables and pick only the best results during filtering (i.e., the highest or lowest based on predicted metric).

\subsection{Table Segmentation}

One goal of table segmentation is to generalise to tables from unseen tasks. To study this, we partitioned SEGMENTEDTABLES dataset into 11 folds, based on the task name extracted from paper abstracts. The fold with tables from Image Classification papers is always used as a validation set. For each of the remaining 10 folds we train 5 models with a given fold used as a test set and the other 9 folds used as training data. The final table segmentation model used in AXCELL is the one with the highest micro $F_{1}$ score on the validation set.

Table 3 shows micro precision, recall and $F_{1}$ score of classifying each non-numeric cell into one of 5 exclusive classes: dataset, metric, competing model, paper's model or other.

We can see that we achieve strong results on all tasks, although some tasks perform better than others. A task like semantic segmentation has less table and benchmark diversity, so benchmark tables for datasets like Cityscapes and PASCAL VOC 2012 are fairly standardised across papers. This makes extraction fairly straightforward. In contrast, the worse performing tasks are unusual in their own way. In image generation, for instance, we are less able to extract the correct dataset entity, whereas in speech recognition, our model has more problems distinguishing paper models from competing models; see Figure 6 in the Appendix.

\subsection{Linking}

To evaluate linking performance in isolation of other steps we run it on tables with ground truth type and segmentation annotations. The annotations are available in the SEgmentedTABLES dataset for 24 Speech Recognition and 32 Semantic Segmentation papers with 287 annotated leaderboard records in total. For each cell with associated leaderboard annotation we generate cell contexts and use linking to retrieve the top-5 predictions. We test four approaches to generate evidence of mentions. 
Table 3: Table segmentation results for 10-fold training with image classification papers fixed as a validation set and variable test set. Micro precision, recall and $F_{1}$ score are averaged over 5 runs.

\begin{tabular}{lrrrrrrr}
\hline & \multicolumn{3}{c}{ validation } & & \multicolumn{3}{c}{ test } \\
\cline { 2 - 3 } \cline { 8 - 8 } test set & $\mathrm{P}$ & $\mathrm{R}$ & $\mathrm{F}_{1}$ & & $\mathrm{P}$ & $\mathrm{R}$ & $\mathrm{F}_{1}$ \\
\hline image gen. & 84.5 & 87.9 & 86.2 & 73.4 & 81.6 & 77.3 \\
misc. & 84.0 & 88.2 & 86.0 & & 81.7 & 93.5 & 87.2 \\
machine trans. & 83.1 & 90.8 & 86.8 & & 80.5 & 94.4 & 86.9 \\
NLI & 83.6 & 89.6 & 86.5 & 84.5 & 97.3 & 90.4 \\
object detection & 81.9 & 91.4 & 86.3 & & 83.7 & 96.7 & 89.7 \\
pose estimation & 85.1 & 89.9 & 87.4 & & 86.0 & 96.8 & 91.1 \\
question ans. & 83.6 & 89.5 & 86.4 & 80.4 & 89.6 & 84.8 \\
semantic seg. & 81.4 & 91.1 & 86.0 & & 90.2 & 95.9 & 92.9 \\
speech rec. & 84.7 & 89.8 & 87.2 & & 67.2 & 90.7 & 77.1 \\
text class. & 83.9 & 90.4 & 87.0 & 74.9 & 93.3 & 83.1 \\
\hline
\end{tabular}

Bag-of-Phrases The full name and any word (which is not an English stop-word) occurring in the name of a metric or dataset (as found in taxonomy) is evidence of mention. For example, for Exact Match Ratio metric we get exact match ratio, exact, match and ratio.

Abbreviations We run an abbreviation detector (Neumann et al., 2019) over the ARXIVPAPERS dataset to extract pairs of common abbreviations and their full forms. The previous approach is extended with abbreviations of full forms occurring in the name of the metric or dataset. For example, with an extracted abbreviation-full form pair (en-vi, English-Vietnamese) and dataset name IWSLT2015 English-Vietnamese, en-vi is added as mention evidence for this dataset. For the Exact Match Ratio metric we extend the Bag-of-Phrases evidence with: em and er (extracted Exact Match Ratio abbreviations), em (extracted Exact Match abbreviation), $m r$ (extracted Match Ratio abbreviation) and $r$ (extracted Ratio abbreviation). To deal with the noise in abbreviations for a given full form we include only short forms that appear at least $20 \%$ of times as an abbreviation of that full form.

Manually Curated We extend the Bag-ofPhrases approach with list of manually curated mention evidence. Only mentions of datasets and metrics related to speech recognition and semantic segmentation are modified.

Combined The previous approach extended with abbreviations.

In Table 4 we show Top- 1 and Top-5 accuracy of the predictions over all leaderboard records from
Table 4: Linking performance using ground truth annotations of table types and segmentation.

\begin{tabular}{|c|c|c|c|c|c|c|c|c|}
\hline \multirow{3}{*}{ evidence } & \multicolumn{8}{|c|}{ Top-1 Accuracy [\%] } \\
\hline & \multicolumn{4}{|c|}{ speech rec. } & \multicolumn{4}{|c|}{ sem. segmentation } \\
\hline & TDMS & $\mathrm{T}$ & $\mathrm{D}$ & M & TDMS & $\mathrm{T}$ & $\mathrm{D}$ & M \\
\hline $\mathrm{BoP}$ & 42 & 86 & 45 & 72 & 49 & 95 & 71 & 67 \\
\hline abbrs & 56 & 87 & 57 & 74 & 56 & 95 & 79 & 74 \\
\hline curated & 76 & 87 & 77 & 87 & 77 & 95 & 89 & 87 \\
\hline \multirow[t]{2}{*}{ combined } & 67 & 87 & 68 & 78 & 72 & 95 & 86 & 85 \\
\hline & \multicolumn{8}{|c|}{ Top-5 Accuracy [\%] } \\
\hline \multirow{2}{*}{ evidence } & \multicolumn{4}{|c|}{ speech rec. } & \multicolumn{4}{|c|}{ sem. segmentation } \\
\hline & TDMS & $\mathrm{T}$ & $\mathrm{D}$ & M & TDMS & $\mathrm{T}$ & & M \\
\hline $\mathrm{BoP}$ & 72 & 88 & 73 & 84 & 82 & 99 & 89 & 93 \\
\hline abbrs & 76 & 89 & 76 & 84 & 93 & 100 & 94 & 99 \\
\hline curated & 85 & 90 & 85 & 91 & 97 & 99 & 99 & 99 \\
\hline combined & 81 & 89 & 81 & 89 & 97 & 99 & 99 & 99 \\
\hline
\end{tabular}

each collection of papers. Using abbreviations significantly improves the performance over the Bag-of-Phrases approach. The worse performance caused by adding abbreviations to manually curated lists suggests that abbreviations could increase the rate of false-positive matches of mentions. Another explanation might be that manually curated lists of mentions are biased towards leaderboards related to speech recognition and semantic segmentation due to construction of the lists.

The overall performance of the linking step allows us to use it in production environment for efficient semi-automated extraction of results. Our solution proposes to users the Top-5 predictions associated with cells they indicated, thus eliminating the tedious and error-prone step of matching the results with existing leaderboards and ensuring that metric values are correctly normalized.

\subsection{End-to-End Performance}

We use annotations for Semantic Segmentation papers from SEgmentedTABles and LinkeDRESUlts datasets to analyse how AxCELl performs in an end-to-end fashion. Figure 4 shows fractions of gold truth records incorrectly rejected in various steps of our pipeline. Both table type classification and segmentation steps were done using models trained with the Semantic Segmentation fold as a test set.

The most common reason for misprediction of datasets is confusion between validation and test sets. Additionally the linking model has difficulties in distinguishing between variants of Intersection over Union metrics (mean IoU, frequency weighted IoU, class and category IoU). The confus- 


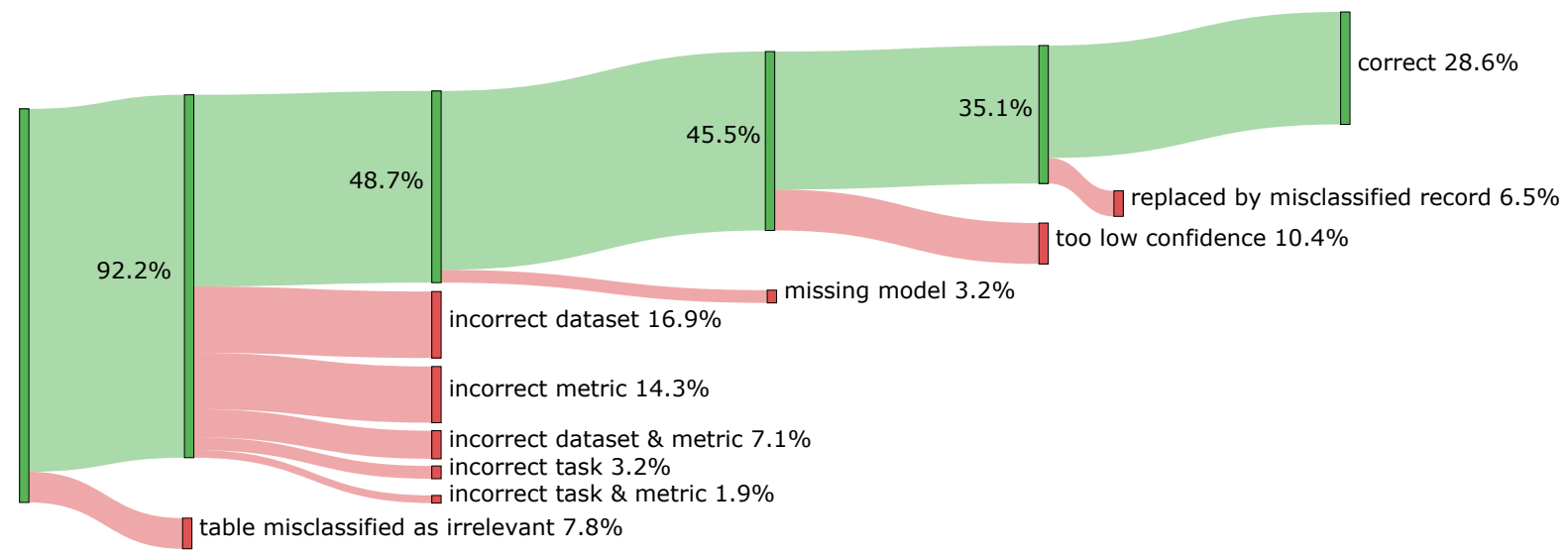

Figure 4: Analysis of end-to-end extraction on the Semantic Segmentation fold of LiNKEDRESULTS dataset. Green blocks show percentage of gold truth tuples of (task, dataset, metric, score) that are correctly classified in various stages of our pipeline. Red blocks show reasons for which a given fraction of gold truth records is incorrectly rejected.

ing datasets and metrics names are also reasons for a low confidence of linked results, as the score is distributed over similar entities.

One should keep in mind that the above analysis might not fully generalise to other tasks. As shown in Table 3 and Figure 6, table segmentation performs differently on papers related to different machine learning tasks. Moreover, it is more common in case of Semantic Segmentation papers to report results on both validation and test sets due to test sets often being hidden. The difference between tasks is also apparent in linking performance on Speech Recognition and Semantic Segmentation papers, as presented in Table 4. While the Top-1 Accuracy is similar for both tasks, in terms of Top5 Accuracy the linking step performs significantly better on Semantic Segmentation papers-most of the time the top 5 entries are sufficient to cover variants of Semantic Segmentation datasets and metrics.

\section{Future Work}

We cover three possible extensions to our work for future research.

First, we might want to consider methods that retrieve all results rather than just the principal results introduced in the paper. This includes extracting ablation studies to enable search over fine-grained comparison results.

Secondly, we could look more into automatic taxonomy discovery. Currently, we assume a closeddomain approach with a taxonomy of leaderboards known in advance. While manually extending the taxonomy requires only adding the task, dataset and metric names, it becomes problematic to cover a large fraction of papers due to publication rate and long tail of leaderboards.

Finally, to relax the necessity of AxCELL to have access to $\mathrm{LTT}_{\mathrm{E}} \mathrm{X}$ source we consider using the ARXIVPAPERS dataset as a corpus to train extraction working directly with PDF files.

\section{Conclusions}

We presented a pipeline for extracting results from machine learning papers. Our method performs well across various tasks and leaderboards within machine learning, with a taxonomy that can be easily extended without retraining. Additionally we released a new collection of datasets for training and evaluating on the results extraction task. These datasets enable the training of more fine-grained feature extractors and detailed error analysis. We demonstrated that our approach achieves significant performance gains over the state-of-the-art. Future work may want to build on our approach for more comprehensive extraction tasks, focussing on more types of result, as well as other information contained in papers such as architectural details and hyperparameters.

\section{Acknowledgements}

The authors would like to thank Waleed Ammar, Sebastian Kohlmeier, Iz Beltagy, and Adam Liska for useful discussion and feedback. 


\section{References}

Jacob Devlin, Ming-Wei Chang, Kenton Lee, and Kristina Toutanova. 2019. BERT: Pre-training of deep bidirectional transformers for language understanding. In Proceedings of the 2019 Conference of the North American Chapter of the Association for Computational Linguistics: Human Language Technologies, Volume 1 (Long and Short Papers), pages 4171-4186, Minneapolis, Minnesota. Association for Computational Linguistics.

Majid Ghasemi-Gol and Pedro A. Szekely. 2018. Tabvec: Table vectors for classification of web tables. CoRR, abs/1802.06290.

Jonathan Herzig, Pawel Krzysztof Nowak, Thomas Müller, Francesco Piccinno, and Julian Eisenschlos. 2020. TaPas: Weakly supervised table parsing via pre-training. In Proceedings of the 58th Annual Meeting of the Association for Computational Linguistics, pages 4320-4333, Online. Association for Computational Linguistics.

Yufang Hou, Charles Jochim, Martin Gleize, Francesca Bonin, and Debasis Ganguly. 2019. Identification of tasks, datasets, evaluation metrics, and numeric scores for scientific leaderboards construction. In Proceedings of the 57th Annual Meeting of the Association for Computational Linguistics, pages 5203-5213, Florence, Italy. Association for Computational Linguistics.

Jeremy Howard and Sylvain Gugger. 2020. fastai: A layered API for deep learning. Information, 11.

Jeremy Howard and Sebastian Ruder. 2018. Universal language model fine-tuning for text classification. In Proceedings of the 56th Annual Meeting of the Association for Computational Linguistics (Volume 1: Long Papers), pages 328-339, Melbourne, Australia. Association for Computational Linguistics.

Taku Kudo. 2018. Subword regularization: Improving neural network translation models with multiple subword candidates. In Proceedings of the 56th Annual Meeting of the Association for Computational Linguistics (Volume 1: Long Papers), pages 66-75, Melbourne, Australia. Association for Computational Linguistics.

Nikola Milosevic, Cassie Gregson, Robert Hernandez, and Goran Nenadic. 2019. A framework for information extraction from tables in biomedical literature. International Journal on Document Analysis and Recognition (IJDAR).

Tom Mitchell. 2006. The discipline of machine learning. Machine Learning Department technical report CMU-ML-06-108, Carnegie Mellon University.

Mark Neumann, Daniel King, Iz Beltagy, and Waleed Ammar. 2019. ScispaCy: Fast and robust models for biomedical natural language processing. In Proceedings of the 18th BioNLP Workshop and Shared Task, pages 319-327, Florence, Italy. Association for Computational Linguistics.
David Pinto, Andrew McCallum, Xing Wei, and W. Bruce Croft. 2003. Table extraction using conditional random fields. In Proceedings of the 26th Annual International ACM SIGIR Conference on Research and Development in Informaion Retrieval, SIGIR '03, page 235-242, New York, NY, USA. Association for Computing Machinery.

Sebastian Ruder. 2018. Tracking the Progress in Natural Language Processing.

Mayank Singh, Rajdeep Sarkar, Atharva Vyas, Pawan Goyal, Animesh Mukherjee, and Soumen Chakrabarti. 2019. Automated early leaderboard generation from comparative tables. In Advances in Information Retrieval - 41st European Conference on IR Research, ECIR 2019, Cologne, Germany, April 14-18, 2019, Proceedings, Part I, volume 11437 of Lecture Notes in Computer Science, pages 244-257. Springer.

Richard Socher, Alex Perelygin, Jean Wu, Jason Chuang, Christopher D. Manning, Andrew Ng, and Christopher Potts. 2013. Recursive deep models for semantic compositionality over a sentiment treebank. In Proceedings of the 2013 Conference on Empirical Methods in Natural Language Processing, pages 1631-1642, Seattle, Washington, USA. Association for Computational Linguistics.

Mingxing Tan and Quoc V. Le. 2019. Efficientnet: Rethinking model scaling for convolutional neural networks. ICML.

Xing Wei, Bruce Croft, and Andrew Mccallum. 2006. Table extraction for answer retrieval. Inf. Retr. 9(5):589-611. 
Table 5: ULMFiT language model architecture and hyperparameters.

\begin{tabular}{lr}
\hline vocabulary size & 30,000 \\
tokenization & unigram model \\
RNN type & LSTM \\
recurrent layers & 3 \\
embeddings dimension & 400 \\
hidden state dimension & 1152 \\
\hline optimizer & AdamW \\
lr schedule & one cycle policy \\
maximum lr & 0.01 \\
weight decay & 0.1 \\
pretraining & 12 epochs \\
batch size & 256 \\
BPTT & 80 \\
number of parameters & $32 \mathrm{M}$ \\
floating-point arithmetic & $\mathrm{fp} 32$ \\
\hline
\end{tabular}

\section{Appendix}

\section{A Training Details}

\section{A.1 Language Model Pre-training}

Table Type Classifier and Table Semantic Segmentation models use ULMFiT architecture (Howard and Ruder, 2018) with a language model pretrained from scratch on the ARXIVPAPERS dataset. We trained a single language model with most of the hyperparameters set to the defaults from the fast.ai implementation (Howard and Gugger, 2020) (see Table 5).

All ULMFiT-based models were trained on a single NVIDIA Tesla V100 GPU instance with 16 GB of memory. See the corresponding Jupyter notebooks for the average training times.

\section{A.2 Table Type Classifier}

We use the pre-trained language model described above to train Table Type Classifier on the SEGMENTEDTABLES dataset. We used the Image Classification fold of the SEGMENTEDTABLES dataset as a validation set, Speech Recognition fold as a test set and the remaining 9 folds as a training set. We run grid search over configurations presented in Table 6. The model with the best binary accuracy (distinguishing leaderboard and ablation tables from irrelevant tables) on the validation set is used in AXCELL. All models are trained for 12 epochs in total with gradual unfreezing of encoder layers.
Table 6: ULMFiT table classifier hyperparameters. Multiple values were used for grid search, with the same 5 random seeds per configuration. The final configuration is in bold.

\begin{tabular}{lr}
\hline dropout mult. & {$[0.0,0.5, \ldots, \mathbf{1 . 0}]$} \\
batch size & {$[64, \mathbf{1 2 8}, 192,256]$} \\
floating-point & {$[\mathrm{fp} 16, \mathbf{f p 3 2}]$} \\
validation set & Image Classification \\
test set & Speech Recognition \\
features & {$[$ caption, caption+headers] } \\
\hline
\end{tabular}

Table 7: ULMFiT table semantic segmentation hyperparameters. Multiple values were used for grid search, with the same 5 random seeds per configuration. The final configuration is in bold.

\begin{tabular}{lr}
\hline mask query & {$[$ False, True $]$} \\
lowercase input & {$[$ False, True $]$} \\
dropout mult. & {$[0.0,0.5, \ldots, \mathbf{0 . 7 5}, \ldots, 1.0]$} \\
batch size & $\mathbf{6 4}$ \\
floating-point & $\mathbf{f p 1 6}$ \\
validation set & Image Classification \\
test set & {$[\ldots$, Pose Estimation, ... $]$} \\
\hline
\end{tabular}

\section{A.3 Table Semantic Segmentation}

We use the pre-trained language model and folds of the SEgmentedTables dataset. We used the Image Classification fold as a validation set. For each of the remaining 10 folds we run grid search with a given fold used as a test set and the other 9 folds used as training data. The search was performed over the configurations showed in Table 7. The model with the best micro $F_{1}$ score on the validation set is used in AxCELL. Table 8 presents features input to the model. All models are trained for 10 epochs in total.

Table 8: Features For Table Segmentation

\begin{tabular}{|c|c|}
\hline Feature & Description \\
\hline $\begin{array}{l}\text { is emphasised } \\
\text { cell style } \\
\text { text }\end{array}$ & $\begin{array}{l}\text { whether text in cell is bold, colored, etc. } \\
\text { e.g. "align-left top-border" } \\
\text { mentions of cell's content (as in Fig- } \\
\text { ure 3) }\end{array}$ \\
\hline cell content & $\begin{array}{l}\text { cell's content without styles and refer- } \\
\text { ences, e.g. "ULMFiT" }\end{array}$ \\
\hline row context & $\begin{array}{l}\text { concatenated cell's row, e.g. "ULMFiT } \\
<\text { sep }>94.5 \%<\text { sep }>92.1 \% "\end{array}$ \\
\hline column context & $\begin{array}{l}\text { concatenated cell's column, e.g. } \\
\text { "Method <sep> LSTM <sep> GRU } \\
\text { <sep> ULMFiT < <ep> BERT" }\end{array}$ \\
\hline cell reference & $\begin{array}{l}\text { list of reference ids used in cell, e.g. } \\
\text { "bib4, bib18" }\end{array}$ \\
\hline
\end{tabular}


Table 9: Linking and filtering hyperparameters.

\begin{tabular}{|c|c|}
\hline$p(n \mid$ Task, Paper $)$ & 0.1 \\
\hline$p(n \mid$ Task, Abstract $)$ & 1.0 \\
\hline$p(n \mid$ Task, Sections $)$ & 1.0 \\
\hline$p(n \mid$ Task, Caption $)$ & 0.1 \\
\hline$p(n \mid$ Task, Table $)$ & 0.1 \\
\hline$p(n \mid$ Dataset, Paper $)$ & 0.99 \\
\hline$p(n \mid$ Dataset, Abstract $)$ & 1.0 \\
\hline$p(n \mid$ Dataset, Sections $)$ & 1.0 \\
\hline$p(n \mid$ Dataset, Caption $)$ & 0.25 \\
\hline$p(n \mid$ Dataset, Table $)$ & 0.01 \\
\hline$p(n \mid$ Metric, Paper $)$ & 0.99 \\
\hline$p(n \mid$ Metric, Abstract $)$ & 1.0 \\
\hline$p(n \mid$ Metric, Sections $)$ & 1.0 \\
\hline$p(n \mid$ Metric, Caption $)$ & 0.25 \\
\hline$p(n \mid$ Metric, Table $)$ & 0.01 \\
\hline$p(m \mid n$, Task $)$ & 0.01 \\
\hline$p(m \mid n$, Dataset $)$ & 0.001 \\
\hline$p(m \mid n$, Metric $)$ & 0.01 \\
\hline filtering threshold ${ }_{1}$ & 0.8 \\
\hline filtering threshold ${ }_{2}$ & 0.85 \\
\hline
\end{tabular}

\section{A.4 Linking and Filtering}

Table 9 shows manually tuned hyperparameters for linking and filtering. The results with confidence score in $\left[\right.$ threshold $_{1}$, threshold 2 ) are not returned, but can prevent returning inferior results (in terms of metric value).

\section{B Datasets}

\section{B.1 ArXIVPaPers Dataset}

The ARXIVPAPERS dataset consists of 104,710 papers published on arXiv.org in the following categories: Artificial Intelligence (cs.AI), Computation and Language (cs.CL), Computer Vision and Pattern Recognition (cs.CV), Information Retrieval (cs.IR), Machine Learning (stat.ML, cs.LG), Neural and Evolutionary Computing (cs.NE).

When submitting a preprint to arXiv.org the submitter must either ${ }^{7}$ grant arXiv.org a non-exclusive and irrevocable license to distribute the article 8 or select one of CC BY 4.0, CC BY-SA 4.0, CC BY-NC-SA 4.0 or CC0 1.0 public domain license. Currently the most common is the first, default op-

/https://arxiv.org/help/license

8 http://arxiv.org/licenses/ nonexclusive-distrib/1.0/license.html
Table 10: Statistics of the NLP-TDMS (Hou et al. 2019) Full and Exp datasets.

\begin{tabular}{lrr}
\hline & Full & Exp \\
\hline unique leaderboards & 168 & 77 \\
unique tasks & 35 & 18 \\
unique datasets & 99 & 44 \\
unique metrics & 72 & 30 \\
\hline papers & 332 & 332 \\
results & 848 & 606 \\
\hline
\end{tabular}

tion. Additionally, arXiv.org provided metadata of submitted papers is available in public domain.

As a consequence of legal requirements we are not able to fully publish the dataset of articles in a ready to use form, with extracted texts and tables. In order to make research in this area reproducible and results comparable, we publish our extraction pipeline and detailed information of extraction results. In particular, each paper contained in the ARXIVPAPERS dataset includes the following fields:

- arxiv_id: arXiv identifier with version,

- archive_size: the file size in bytes of the eprint archive,

- sha256: SHA-256 hash of the e-print archive,

- title: paper's title,

- status: the text and tables extraction status for this paper, one of: success, no-tex (LaTeX source is unavailable), processing-error (extraction issues), withdrawn (the paper is withdrawn from arXiv),

- sections: number of extracted sections and subsections,

- tables: number of extracted tables.

Extraction of texts and tables from papers was run on a single machine with 48 cores / 96 threads CPU with $2.5 \mathrm{GHz}$ base clock. See the corresponding Jupyter notebooks for the average extraction time.

\section{B.2 SegmentedTables and LINKEDRESULTS datasets}

The SegmentedTables dataset contains annotations of 1,994 tables. Each paper contains the following fields: 
Table 11: Statistics for the SEgmentedTables and LINKEDRESULTS datasets.

\begin{tabular}{lr}
\hline \multicolumn{2}{c}{ SEGMENTEDTABLES } \\
\hline papers & 352 \\
tables & 1994 \\
leaderboard tables & 796 \\
ablation tables & 468 \\
\hline \multicolumn{2}{c}{ LINKEDRESULTS } \\
\hline unique leaderboards & 470 \\
unique tasks & 56 \\
unique datasets & 245 \\
unique metrics & 88 \\
\hline papers & 239 \\
results & 1591 \\
\hline
\end{tabular}

- arxiv_id: arXiv identifier with version,

- sha256: SHA-256 hash of the e-print archive,

- fold: one of 11 folds (image classification, image generation, machine translation, miscellaneous, natural language inference, object detection, pose estimation, question answering, semantic segmentation, speech recognition, text classification), assigned automatically based on tasks names found in paper's abstract,

- tables: annotated tables with

- index: 0-based index of tables extracted from paper,

- leaderboard: a boolean denoting if this table is a leaderboard table,

- ablation: a boolean denoting if this table is an ablation table,

- dataset_text: datasets mentioned in table's caption, not normalized,

- segmentation: for leaderboard tables, a 2D array (list of lists) with one label per cell.

Additionally we annotated a subset of the tables present in SEGMENTEDTABLES with performance results. Each table has an array of records with items containing the following fields:

- task, dataset, metric: task, dataset and metric names normalized across all papers from the dataset,
- value: normalized metric value,

- model: model name,

- row, column: 0-based cell location with this result.

Annotation Process Both datasets were annotated in our custom made web interface. For each paper the annotator is present with: title, abstract, tags (user editable), notes (user editable) and extracted tables. The interface allows annotators to quickly consult: PDF version of the paper, HTML version of the paper, Papers With Code and Semantic Scholar pages of the paper.

For each table extracted from the paper we show:

- caption (extracted),

- dataset text (user editable): caption fragment denoting datasets presented in the table,

- notes (user editable),

- tags (user editable),

- table content with color-coded segmentation.

Dataset text field denotes comma separated mentions of datasets found in table's caption. For example, for caption "Table 8: WER on SWB and $\mathrm{CH}$ with various $\mathrm{LM}$ configurations." the annotators were instructed to put "SWB, CH", i.e., to use exact form from the caption and not the full dataset name.

Table tags are defined as follows:

- leaderboard: table contains the principal results of the paper, including comparisons with other papers,

- ablation: table compares different variants of the paper's methodology,

- error: parsing error in the table extraction,

- datasets: table describing datasets used in the paper,

- architecture: table listing hyperparameters or architecture details,

- irrelevant: other type of tables, f.e., showing samples from a dataset. 
The SegmentedTables dataset contains tables not tagged with error label. Table tags are not exclusive.

For semantic segmentation, tables are present as a grid. An annotator can select a range of cells and assign them one of the following classes:

- best model: the best performing model introduced in the paper being annotated,

- paper model: model introduced in the paper that is not the best performing,

- competing model: model from another paper used for comparison or a baseline method used by authors,

- subdataset: subdataset (f.e., "dev", "test" or "MS-COCO Trees",

- dataset,

- paper dataset: dataset introduced in the paper

- metric,

- error: parsing issue, not required if the table is tagged with the error tag,

- parameters: model parameters used to distinguish various configurations (f.e., number of parameters, hidden state size, backbone network),

- meta: cell describing what is in other cells, f.e., "Model", "Dataset", "Task", "Our models".

Segmentation annotation was done for tables labelled with the leaderboard tag. In order to easy present the color-coded table structure the cell tags are exclusive. For cells for which more than one tag applies, the annotators were instructed to use most informative tag. For example, a cell containing "TIMIT PER" should be tagged as "dataset" and not "metric", as metric is often implied by dataset.

By selecting cells an annotator can annotate what is in corresponding cells by editing a dynamically created spreadsheet-like grid. The grid allows one to specify: task name, dataset name, metric name, metric value and model name. The annotators were instructed to provide records only for cells corresponding to the best performing models introduced in a given paper.

The interface allows to link directly to a particular table to make it easy for annotators to consult ambiguous cases.
Table 12: Statistics for the PWC LEADERBoARDS dataset with all entries (Full) and entries restricted to our taxonomy (Restricted).

\begin{tabular}{lrr}
\hline & Full & Restricted \\
\hline unique leaderboards. & 2295 & 649 \\
unique tasks & 252 & 134 \\
unique datasets & 1156 & 433 \\
unique metrics & 414 & 162 \\
\hline papers & 733 & 516 \\
results & 5406 & 2802 \\
\hline
\end{tabular}

Figure 5: A screenshot of the labeling interface used to annotate PWC LEADERBOARDS dataset. An annotator is presented with tables extracted from a paper on the left-hand side and annotations on the right hand side.

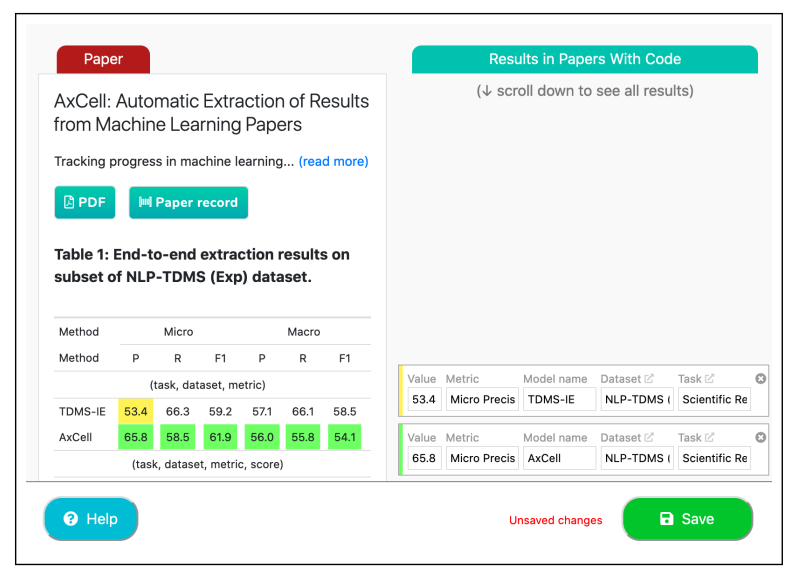

\section{B.3 PWC LEADERBOARDS dataset}

The PWC LEADERBOARDS dataset is based on open data published by Papers With Code and annotated by their community. We converted the data into format similar in structure to the LINKEDRESULTS dataset.

\section{Mention Probabilities}

Using the methodology from Section 3.4, we can calculate $p(k \mid E)$ by combining probabilities of mentions, $p(m \mid \neg n, t$, property $(t, k))$.

We compute all possible mentions directly from tasks, datasets and metrics names appearing in leaderboards. For a name of dataset or metric the mentions list consists of the whole name as well as each word, without duplicates and English stop words. As tasks names often consist of common words, to limit the number of false positives the mentions list for a given task contains only that task's name. The mentions can be additionally extended with human curated lists or abbreviations 
extracted from papers, as described in Section 6.3

Let $R(t)=\{\operatorname{property}(t, k): k \in\{1, \ldots, K\}\}$ be a set of all entities of type $t$ and let $M(t, r)$ denote a set of all possible mentions for a given entity $r \in R(t)$. We compute the probability $p(m \mid \neg n$, property $(t, k))$ assuming all mentions (separately for tasks, datasets and metrics) for a given entity $r$ are distributed uniformly, $p(r \mid \neg n, t, m)=1 /|M(t, r)|$. We then use Bayes rule to get $p(m \mid \neg n$, property $(t, k))$, assuming that all mentions of a given type are distributed uniformly. This results in the conditional probability of a mention being inversely proportional to the number of entities having that mention evidence in common:

$$
\begin{aligned}
& p(m \mid \neg n, t, \operatorname{property}(t, k)) \propto \\
& \frac{1}{\left|\left\{r^{\prime} \in R(t): m \in M\left(t, r^{\prime}\right)\right\}\right|} .
\end{aligned}
$$

\section{Additional Results}

Figure 7: Confusion matrix of table type classification step.

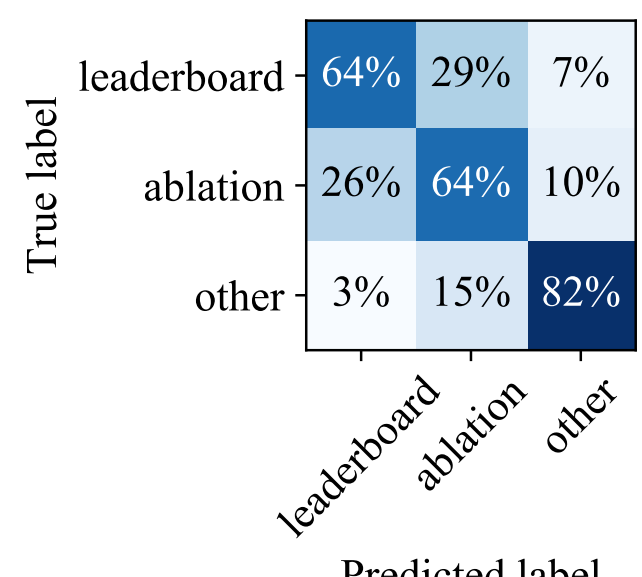

Figure 6: Confusion matrices of segmenting cells into five classes: dataset (including subdatasets), metric, model introduced in processed paper, competing model and other. Results averaged over 5 runs for each task, using 10-fold training as described in Section 6.2 with tables from a) Speech Recognition, b) Image Generation and c) Semantic Segmentation papers as a test set.

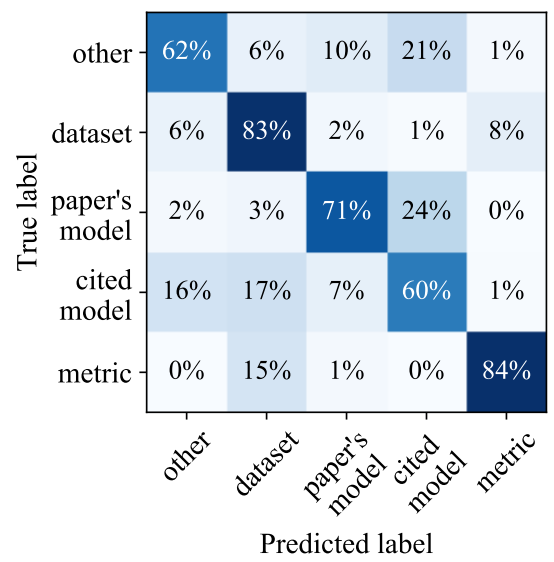

(a) Speech Recognition

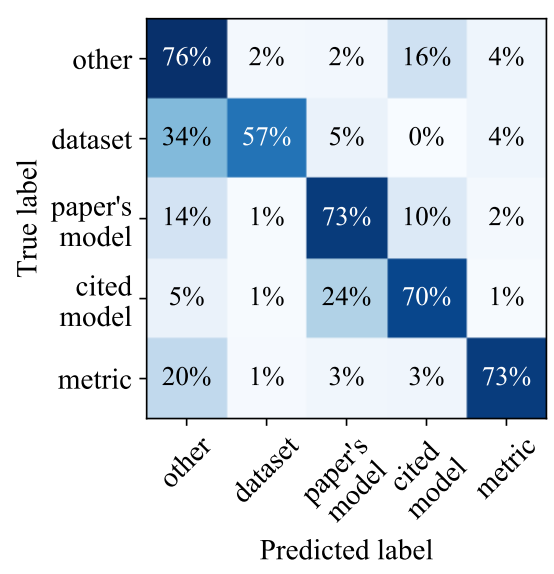

(b) Image Generation

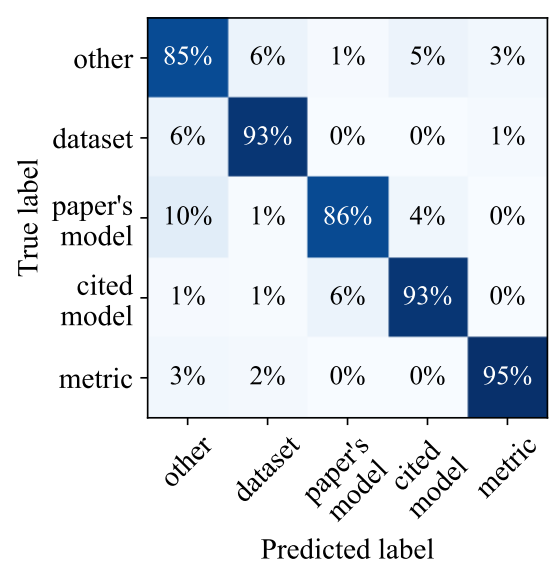

(c) Semantic Segmentation 